\title{
PONTOS CRÍTICOS DE IMPACTO EM LINHAS DE BENEFICIAMENTO UTILIZADAS PARA CITROS NO ESTADO DE SÃO PAULO ${ }^{1}$
}

\author{
MARCOS DAVID FERREIRA2 ${ }^{2}$ MICHELE CARVALHO DA SILVA ${ }^{3}$, GUSTAVO GARCIA DE TOLEDO \\ CAMARGO $^{4}$, LILIAN AMORIM ${ }^{5}$, IVAN HERMAN FISCHER 6 .
}

\begin{abstract}
RESUMO - A produção citrícola brasileira é uma das mais importantes para a fruticultura do País, comercializando principalmente para o mercado externo. O impacto mecânico é um fator importante a ser minimizado, visando a reduzir as perdas pós-colheita, diminuindo consequientemente os prejuízos decorrentes do manejo inadequado. Neste trabalho, foram avaliados os pontos críticos e o nível de impacto em que os frutos são submetidos em equipamentos nacionais e importados, utilizados para beneficiamento e classificação de citros. Utilizouse da esfera instrumentada $(70 \mathrm{~mm}$ ) (Techmark, Inc., Lansing, EUA), com registrador de aceleração, para a avaliação da magnitude de impactos $\left(\mathrm{G}, \mathrm{m} / \mathrm{s}^{2}\right)$ nos pontos de transferência das linhas de classificação de laranjas e lima-ácida Tahiti. A esfera instrumentada foi colocada, juntamente com os frutos, na etapa de recebimento das linhas que funcionavam em seu volume padrão diário, e seguiu o fluxo dos frutos até a etapa de classificação. Os resultados demonstraram que os maiores impactos foram causados por quedas em pontos de transferência com superfícies rígidas, sendo os maiores valores encontrados na etapa de recebimento e na entrada dos frutos em "containers" utilizados para o armazenamento. Observou-se, também, que a magnitude de impacto foi significativamente reduzida quando se utilizaram superfícies recobertas com acolchoados nos diversos pontos de transferência.
\end{abstract}

Termos para indexação: esfera instrumentada, classificação, lima-ácida, laranja.

\section{EVALUATION OF CRITICAL POINTS OF IMPACT ON CITRUS SORTING LINES IN THE STATE OF SÃO PAULO}

\begin{abstract}
The Brazilian citrus chain is one of the leaders on the export process. Mechanical injury is one important factor that should be minimized on this chain, in order to reduce and decrease postharvest losses due to no appropriate handling. On this work, critical points of impact were evaluated in imported and national equipments used for sorting citrus. The instrumented sphere, 70mm, (Techmark Inc., Lansing, EUA) was used for this evaluation, mesuring the magnitude of impact $\left(\mathrm{G}, \mathrm{m} / \mathrm{s}^{2}\right)$ on transfer points in sorting lines of oranges and lemon. The instrumented sphere was placed together with the fruits in the receiving stage working in its regular daily capacity, following the normal flow until the stage of classification. The results showed that the critical points of impact were at the receiving step and also on the transfer point into the containers for storage. The magnitude of impact was significantly reduced when cushioned surfaces were used on different transfer points. Index terms: instrumented sphere, classification, lemon, orange.
\end{abstract}

Na safra de 2003-2004, o Brasil ocupou a $10^{\text {a }}$ posição no ranking mundial de exportadores de laranja in natura, com a produção de 16.977.767 toneladas (AGRIANUAL, 2005). De acordo com a CONAB (2005), o Brasil chegou a exportar 1.681.221 toneladas de laranjas frescas e processadas em 2003, que aumentou para 1.701.334 toneladas, em 2004.

Vigneault et al. (2002) definiram impactos como movimentos transitórios causados por súbita aceleração ou desaceleração do fruto, causando grandes dissipações de energia, provocando esforços e consequientes danos aos frutos. Deste modo, o impacto mecânico pode provocar ferimentos na casca, tornando o fruto suscetível ao ataque de insetos e doenças. De acordo com SARDI (2005), os principais pontos que favorecem a ocorrência de danos mecânicos, numa linha de beneficiamento, são: queda dos frutos na entrada da linha de beneficiamento; fluxo inadequado; transferência dos frutos entre componentes da linha; superfícies rígidas; velocidades elevadas dos frutos; manutenção inadequada dos equipamentos, e resíduos de cera na linha. Para a avaliação da magnitude de impacto nos pontos de transferência em linhas de beneficiamento e classificação de citros (Miller \& Wagner, 1991) e hortaliças, utilizou-se a esfera instrumentada, mensurando-se a aceleração máxima (AM) $\left(\mathrm{G}=9,81 \mathrm{~m} / \mathrm{s}^{2}\right)$ e mudanças na velocidade $(\triangle \mathrm{v})(\mathrm{m} / \mathrm{s})($ Sargent et al., 1992).

Miller \& Wagner (1991) relatam que 80\% dos impactos descritos em uma linha de classificação para citros variam de 25.G$150 . G$ e são causados por quedas em superfícies rígidas de metal ou em superfícies protegidas por camada de cobertura emborrachada muito fina. Porém, os autores também observaram valores de 300.G, na etapa de recebimento, quando a esfera instrumentada se localizava no topo do "pallet". Sargent et al. (1992) relatam que, reduzindo-se o impacto dos pontos de queda e modificando-se em alguns pontos da linha de classificação de tomates de mesa, com a utilização de protetores de superfície, reduziu-se em até $50 \%$ a pressão de impacto, com conseqüente diminuição nos danos físicos. Timm \& Brown (1991) também relataram diminuição no impacto em linhas de classificação de abacate, mamão e abacaxi, com a utilização de protetores emborrachados.

O objetivo deste trabalho foi identificar os pontos críticos e a magnitude dos impactos em linhas de beneficiamento e classificação de laranjas e limas ácidas localizadas no Estado de São Paulo.

Utilizou-se da esfera instrumentada (Techmark, Inc., Lansing, EUA), equipamento plástico com registrador de aceleração, para a avaliação da magnitude de impactos nos pontos de transferência das linhas de classificação de citros. Os dados armazenados na esfera foram transferidos e analisados em computador, sendo possível a avaliação da magnitude dos impactos a que os frutos foram submetidos nos pontos de transferência das linhas de beneficiamento.

Foram avaliados quatro equipamentos de beneficiamento e classificação de citros, duas linhas para lima-ácida e duas linhas para laranja. Para tanto, foram visitados três galpões de beneficiamento de frutos para exportação, localizados na região central do Estado de São Paulo.

A primeira unidade de beneficiamento analisada era composta

\footnotetext{
${ }^{1}$ (Trabalho 151-2005). Recebido: 20-09-2005. Aceito para publicação: 25-08-2006.

${ }^{2}$ Engenheiro Agrônomo, Pesquisador Colaborador, Faculdade de Engenharia Agrícola UNICAMP, Campinas-SP, marcos.ferreira@agr.unicamp.br, Telefone: $19-3521-1028$

${ }^{3}$ Aluna de Graduação Faculdade de Engenharia Agrícola, UNICAMP, Campinas-SP, michele.silva@agr.unicamp.br.

${ }^{4}$ Engenheiro Agrícola Faculdade de Engenharia Agrícola, UNICAMP, Campinas-SP, gustavogtcamargo@gmail.com.

${ }^{5}$ Engenheira Agrônoma, Professora, Departamento de Fitopatologia, ESALQ/USP, Piracicaba-SP, liamorim@esalq.usp.br.

${ }^{6}$ Aluno de Doutorado, Departamento de Fitopatologia, ESALQ/USP, Piracicaba-SP, ihfische@ carpa.ciagri.usp.br.
} 
por dois equipamentos nacionais para beneficiamento de lima-ácida Tahiti, sendo que, em cada equipamento, se realizava uma fase diferente do beneficiamento e classificação. Em uma primeira fase (A), os frutos eram pré-classificados e recebiam tratamento fitossanitário, sendo submetidos a sete pontos de transferência em uma linha com comprimento de 20 metros; logo após, permaneciam em espera por 24 horas, para então completarem o beneficiamento na segunda fase (B), em uma linha de 70 metros, composta por 9 pontos de transferência. Na linha (A), não ocorria sistema de classificação, e, na linha (B), a classificação era realizada por roletes transversais. $\mathrm{O}$ volume médio processado nestas linhas foi de 6-7 toneladas de frutos por hora.

$\mathrm{Na}$ segunda unidade, foi analisada uma linha de beneficiamento e classificação de laranjas $(\mathbf{C})$ que se caracterizava por ser extensa, com 110 metros de comprimento, totalmente automatizada, desde a recepção dos frutos até a embalagem, composta por equipamentos importados com classificador eletrônico “Optiscan 2000". Nesta linha, eram processados 13,6 toneladas de frutos por hora, sendo estes submetidos a 19 pontos de transferência.

$\mathrm{Na}$ terceira unidade, avaliou-se também uma linha de beneficiamento e classificação de laranjas (D), composta por equipamentos nacionais, com comprimento de $65 \mathrm{~m}$. A linha era automatizada, exceção da recepção e embalagem, e processava 5,1 toneladas de frutos por hora, sendo submetidos a 16 pontos de transferência. Os pontos de transferência, nas quatro linhas avaliadas, interligavam mudanças de processos, os quais eram similares em cada unidade, variando o material utilizado e o comprimento desta. As etapas de limpeza, secagem e polimento são sempre realizadas com escovas, e, na seleção, frutos são expostos em esteiras de roletes. Visando-se a avaliar a redução na aceleração máxima, foi realizado ensaio na linha de beneficiamento D no recebimento de frutos a granel, utilizando-se de superfícies acolchoadas. Para tanto, laranjas foram submetidas à queda de altura de $90 \mathrm{~cm}$ em duas superfícies: colchão de espuma com espessura média de $5 \mathrm{~cm}$ e concreto.

A esfera instrumentada, com limite para o nível de impacto variando entre 15.G e 500.G, foi colocada juntamente com os frutos na etapa de recebimento de cada linha de beneficiamento, que funcionava com sua capacidade normal, seguindo o fluxo dos mesmos até a etapa de classificação. O tempo de permanência da esfera instrumentada, em cada etapa, assim como o tempo para completar o percurso percorrido e os pontos de transferência foram monitorados através de cronômetro de precisão. As medições no

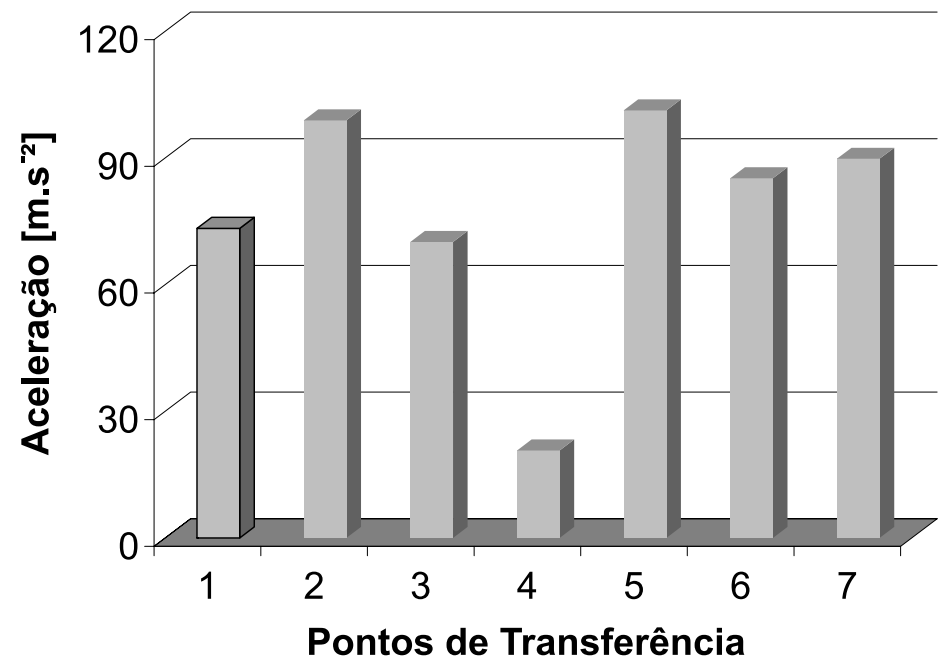

FIGURA 1 - Acelerações médias $(G)$ encontradas na linha de beneficiamento e classificação A, em seus pontos de transferência: 1-Recebimento; 2-Entrada da lavagem; 3-Entrada do classificador; 4-Saída do classificador; 5Saída da seleção; 6-Saída da esteira; 7-Embalagens de recebimento. percurso total da linha de classificação foram repetidas cinco vezes. Após os testes, a esfera instrumentada era removida, e os dados, transferidos para microcomputador. Dados médios de impacto, obtidos nos pontos de transferência de cada unidade de beneficiamento avaliada, foram correlacionados à aceleração máxima (AM) $\left(\mathrm{G}=9,81 \mathrm{~m} / \mathrm{s}^{2}\right)$.

A Figura 1 representa o gráfico das acelerações médias presentes em cada etapa da linha $\mathbf{A}$, utilizada na pré-classificação da lima-ácida Tahiti. Pode-se observar que o nível máximo de impacto ocorreu na etapa 5, que se refere à saída da seleção, com impacto de 101.G. No entanto, observa-se, na Figura 2, que a média de impacto máximo na linha $\mathbf{B}$ ocorreu na etapa 1 , referente à entrada dos frutos na linha, apresentando aceleração média máxima igual a 199.G. Esta

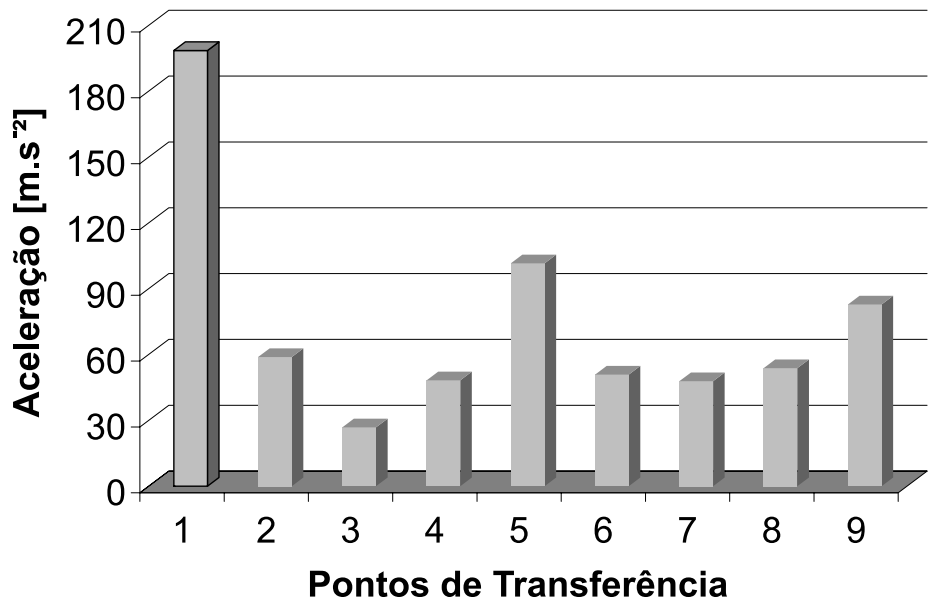

FIGURA 2 - Acelerações médias $(G)$ encontradas na linha de beneficiamento e classificação $\mathbf{B}$, em seus diferentes pontos de transferência: 1-Recebimento; 2-Entrada do secador; 3-Saída do secador; 4-Entrada para aplicação de cera; 5- Saída do secador; 6- Saída da seleção; 7Saída da seleção; 8-Entrada da classificação; 9- Saída da classificação.

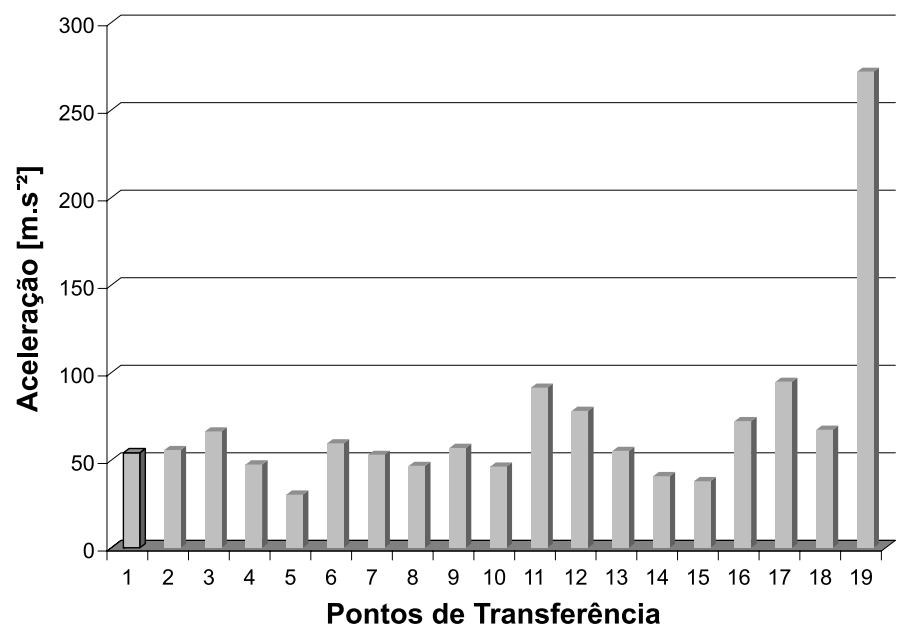

FIGURA 3 - Acelerações médias (G) encontradas na linha de beneficiamento e classificação $\mathbf{C}$, em diferentes pontos de transferência: 1-Recebimento; 2-Lona de entrada; 3Esteira espalhadora; 4-Fim da esteira espalhadora; 5Entrada da lavagem; 6-Entrada da balsa; 7-Esteira (Escolha principal); 8-Saída 1 Esteira (Escolha Principal); 9-Degrau; 10-Saída 2 (Escolha principal); 11-Degrau 1 (Talisca de retorno); 12- Degrau 2 (Talisca de retorno); 13-Entrada da Classificação; 14-Saída da Classificação; 15-Retorno do Classificador; 16-Retorno da Esteira; 17Entrada para Banca de Classificação; 18-Saída da Banca de Classificação; 19-Entrada em containers de recebimento. 


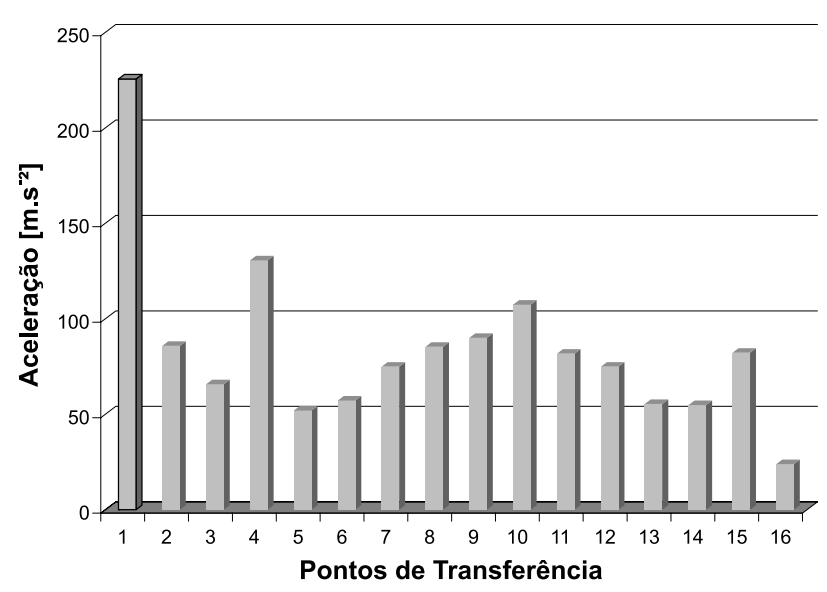

FIGURA 4 - Acelerações médias (G) encontradas na linha de beneficiamento e classificação $\mathbf{D}$, em diferentes pontos de transferência: 1-Recebimento; 2-Entrada da linha (1); 3- Entrada da linha (2); 4-Entrada da pré-classificação; 5-Saída da pré-classificação; 6-Entrada da lavagem; 7Saída da lavagem; 8-Entrada do secador (1); 9-Saída do secador (1); 10-Entrada do secador (2); 11-Saída do secador (2); 12-Entrada da classificação; 13-Saída do rolete classificador; 14-Esteira de separação para classificação; 15-Saída da classificação/entrada da banca; 16- Saída da banca.

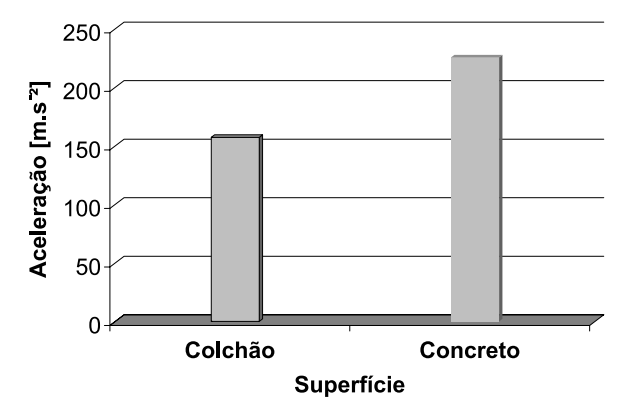

FIGURA 5 - Acelerações médias (G) - Comparação entre superfícies de quedas (Unidade $\mathbf{D}$ ).

variação encontrada entre valores de G., em especial no recebimento, ocorre comumente em etapas realizadas manualmente (Ferreira et al., 2005). O segundo ponto de maior impacto na linha $\mathbf{B}$ ocorreu na Saída do secador, com aceleração média máxima de 102.G. Foi observado que $100 \%$ dos impactos obtidos para linha $\mathbf{A}$ permaneceram na faixa de 21. G. a $101 . \mathrm{G}$, e $88,9 \%$ dos impactos registrados na linha B permaneceram na faixa de 27.G a 102.G, causados principalmente por quedas em superfícies rígidas. Os valores obtidos nestas linhas de beneficiamento indicam melhor desempenho que as linhas de beneficiamento citadas por Miller \& Wagner (1991), onde 80\% dos impactos descritos variavam entre 25.G a 150.G.

Na linha de beneficiamento e classificação C, 94,7\% dos impactos descritos variaram entre 30 e 95.G. A Figura 3 indica que a aceleração máxima chegou a 272.G na etapa 19 , referente à entrada dos frutos nos "containers" utilizados para o armazenamento. Este valor corresponde à situação crítica, onde os primeiros frutos entram na linha de beneficiamento, sofrem queda e conseqüente impacto superior. Embora este valor tenha sido alto, Miller \& Wagner (1991) observaram, em etapas de recebimento, que a aceleração máxima pode atingir 300.G.

Para a linha de beneficiamento e classificação D, 93,7\% dos impactos variaram entre 24.G a 131.G. Na Figura 4, pode-se observar que a etapa 1 , referente ao recebimento nesta linha, apresentou média de aceleração máxima igual a 226.G. Esta etapa consistia no descarregamento dos frutos do caminhão, com queda de $90 \mathrm{~cm}$ de altura, diretamente a uma superfície de concreto. No teste onde a superfície do recebimento era protegida com um colchão de espuma, com o objetivo de amortecer o impacto, observa-se que a média de aceleração diminuiu para 157.G (Figura 5), o que corresponde a uma redução de 30\% no impacto. Timm \& Brown (1991) também relatam um decréscimo no impacto em linhas de classificação de abacate, mamão e abacaxi, quando se utilizaram protetores emborrachados. A diminuição nos valores encontrados, através do uso de materiais amortecedores, é importante, pois, de acordo com Miller \& Wagner (1991), impactos contra superfícies abrasivas, como concreto ou metais, podem ser a causa mais provável de danos aos frutos. Skaria et al. (2003) relatam aumento significativo na incidência de danos físicos em laranjas submetidas ao beneficiamento e classificação em quatro diferentes unidades, quando comparado com frutos retirados diretamente do campo, ou seja, livres de danos mecânicos causados pelo sistema de beneficiamento e classificação. Este aumento nos danos físicos também foi acompanhado por maior presença de doenças e podridões. Observou-se, portanto, que a menor incidência em impacto pode proporcionar diminuição na incidência de danos físicos, influenciando na conservação do produto.

Os pontos de transferência das linhas de beneficiamento e classificação avaliados demonstraram valor máximo de aceleração nas etapas de recebimento e na entrada em "containers" de recebimento, ocorrendo principalmente devido à queda em superfícies rígidas e alturas excessivas. Alternativas de baixo custo, como o uso de superfícies protetoras, ou ainda a redução da altura de queda dos frutos, podem diminuir significativamente a magnitude de impacto e conseqüentemente os danos físicos aos frutos, com maior conservação na pós-colheita.

\section{AGRADECIMENTOS}

À FAPESP, pelo apoio financeiro.

\section{REFERÊNCIAS}

AGRIANUAL 2005: anuário da agricultura brasileira. São Paulo: FNP Consultoria \& Comércio, 2004. p.287-311.

CONAB- Companhia Nacional de Abastecimento. Indicadores da agropecuária. Disponível em: <http://www.conab.gov.br/ download/indicadores/pubindicadores.pdf >. Acesso em: fev. 2005.

FERREIRA, M.D.; FERRAZ, A.C.O ; FRANCO, A.T.O. Tomato packing lines studies with an instrumented sphere in Brazil. Acta Horticulturae, Leuven, v.3, p.1753-1755, 2005.

MILLER, W.M.; WAGNER, C. Florida citrus packing line studies with an instrumented sphere. Applied Engineering in Agriculture, St. Joseph, v.7, n.5, p. 577-581, 1991.

SARDI. Horticulture: Mechanical Injury. South Australian Research and Development Institute, 2001. Disponível em: <http:// www.sardi.sa.gov.au/pages/horticulture/ citrus/hort_citp_ postinjury.htm:sectID=306\&tempID=99>.Acesso em : fev. 2005 .

SARGENT, S. A.; BRECHT, J. K.; ZOELLNER, J. J. Sensitivity of tomatoes at mature-green and breaker ripeness stages to internal bruising. Journal of the American Society for Horticultural Science, Alexandria, v.117, n.1, p.119-123, 1992.

SKARIA, M.; EAYRE, C. G.; MIAO, H.; SOLIS-GRACIA, N.; MACKEY, B. Effect of packing on rot and fruit damage in Rio Red Grapefruit. Subtropical Plant Science, Edinburg, v.55, p.7578, 2003.

TIMM, E. J.; BROWN, G. K. Impacts recorded on avocado, papaya, and pineapple packing lines. Applied Engineering in Agriculture, St. Joseph, v.7, n.4, p.418-422, 1991.

VIGNEAULTC.; BORDINI, M. R.; ABRAHÃO, R.F. Embalagem para frutas e hortaliças. In: CORTEZ, L.A.B.; HONÓRIO, S.L.; MORETTI, C.L. (Ed). Resfriamento de frutas e hortaliças. Brasília: EMBRAPA, 2002.p. 96-121. 